\title{
Research on the influence of theme restaurant interior design on users' consumption behavior based on the layout of interior environment space
}

\author{
Yan Wan \\ Macau University of Science and Technology, Macau 999078, China
}

\begin{abstract}
The traditional research method of user consumption behavior influence is too subjective in the selection of indicators at the time of research, which leads to poor referenceability of research results. To this end, the problems of traditional influence research methods are improved by proposing a method for analyzing the influence of theme restaurant interior design on user consumption behavior based on the spatial layout of the interior environment. On the basis of analyzing user consumption behavior patterns and determining factors affecting consumption behavior, the influence model of user consumption behavior is constructed and statistical indicators are selected to complete the research process. The simulation experiment proves that the research results of applying the method can effectively improve the restaurant operation and have practical value.
\end{abstract}

\section{Introduction}

In order to meet the increasingly high requirements of customers, many restaurants have begun to focus on the personalization of the dining environment, comfortable to build, focusing on the spiritual enjoyment and experience of customers in the dining process. However, with a large number of theme restaurants unevenly into the market, resulting in theme restaurants become a business trend at the beginning of the emergence of many problems. The main restaurant as a commercial space, its interior design has a certain degree of influence on attracting consumer spending. The interior design of commercial space is like a commodity for consumers, carrying information about the characteristics of many goods for people to choose and experience. Space becomes a commodity in consumption, and the commodity must become a symbol to resonate with consumers, so the space atmosphere becomes a symbolic consumption mode. Indoor spatial ambience has a significant influence in the decision-making process and consumption behavior of consumers. For different spatial ambience preferences and importance, solutions are designed to meet the needs of different consumers [1-2]. It is very important for investors, operators, and designers to set a comfortable and safe commercial space design positioning that is favored by the target consumer market.

The method of consumer behavior influence analysis using LMDI theory is mentioned in literature [3], which is mainly through the way of analyzing a large amount of data, and then studying the influence of related factors by extracting the inner laws and characteristics of the data. This method not only has requirements for the comprehensiveness and objectivity of data processing and the method of data inclusion laws processing, but also has more strict criteria for data sources, which leads to more limitations and poor results when this influence analysis method is applied to the subject restaurant interior design as a research object. The analysis method mentioned in the literature [4] is too subjective in the selection of impact analysis related indicators, and the selection of analysis factors is too subjective to achieve the expected results.

In order to quantify this influence, this paper will propose an analysis method using the theory related to the spatial layout of the interior environment, so as to study the influence of theme restaurant interior design on users' consumption behavior.

\section{Research on the influence of theme restaurant interior design on users' consumption behavior based on the layout of interior environment space}

\subsection{Analysis of factors affecting the consumption behavior of theme restaurant users}

\subsubsection{Analysis of user consumption behavior patterns}

Consumer behavior is extremely complex and variable, involving many disciplines, so the study of consumer behavior is also extremely complex. For the consumption behavior of theme restaurant users, the relevant theories

*E-mail: 15901681037@163.com 
of behavioral science can be used to divide. In behavioral science, consumer buying behavior is described and explained as a decision-making process. Parts of this decision-making process can be observed, such as the socio-statistical characteristics of consumers, their buying or non-buying behavior, and the specific products they buy, among others[5]. But the real purchase decision is made in the complex "inner thinking" process of the consumer. According to psychology, human behavior is the brain's response to a stimulus, and in this process, human mental activity governs human behavior. A consumer's purchase of a product is a behavior, which is caused by a certain stimulus, and the purchase is a response to this stimulus. After the external stimulus is received by the consumer, it undergoes a certain psychological process and produces a visible behavioral response, which is the consumer purchase behavior decision process. According to the Howard-Shetch theory shown in figure 1 , the consumption behavior pattern of theme restaurant users is analyzed [6-8].

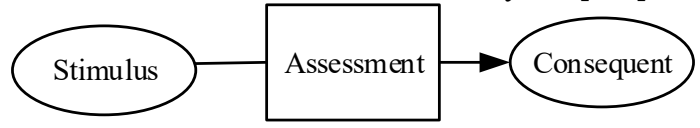

Figure 1 Schematic diagram of Howard-Shetch theory

The Howard-Shetch theory shown in figure 1 consists of three parts: (1) Stimulation: consumers receive information through external stimuli, which come from three sources: physical stimuli, symbolic stimuli and social environment stimuli; (2) Evaluation: it describes how consumers process the impressions formed in their minds after receiving stimuli or information, and how they become willing to buy after adding their own motivation and confidence; (3) Result: after the aforementioned stimuli and evaluation, the final result is that consumers become willing to buy and then generate related consumption behavior.

For theme restaurants, the main difference with other types of restaurants lies in the interior dining environment designed according to the business "theme". Therefore, most of the factors that stimulate the consumption behavior of theme restaurant users are the interior design of the restaurant, and this stimulation is mainly sensory stimulation. We analyze the sensory stimuli that consumers may receive when they consume in theme restaurants, and analyze the factors that influence the consumption behavior of theme restaurant users.

\subsubsection{Determination of factors affecting user consumption behavior}

Based on the above analysis, this paper determines the factors influencing the user consumption behavior of specific theme restaurants from two perspectives: the background elements and design elements of theme restaurants[9].

The light environment can be visually felt by people intuitively and can trigger a host of psychological reactions, and the same is true for the restaurant space as a specific functional space. Different light environments can make people feel different psychological feelings of space, and. Human psychology and food sense have a very subtle and complex relationship. People are not in a static state in the dining space, and the light environment that exists in the room should also change as the line of sight moves. In different environments, the visual contact is different, and the resulting emotional effects are also different [10-12].

According to the above analysis of the specific content, the theme restaurant interior design on the impact of user consumption behavior model is established and through the questionnaire survey the descriptive indicators are analyzed.

\subsection{Descriptive statistics of user consumption behavior}

Based on the above analysis content, combined with the perspective of the effect of indoor environment spatial layout theory on restaurant interior design, and based on Howard-Shetch theory, the influence model of users' consumption behavior is established.

The confidence index is mostly expressed by the correlation coefficient, of which the Crobach $\alpha$ confidence coefficient method is the simplest and most commonly used method, and the magnitude of the coefficient indicates the confidence level of the statistical description of the model, generally speaking, the larger the coefficient the higher the confidence level, and conversely, the smaller the coefficient the lower the confidence level. The formula for calculating the Crobachaconfidence coefficient is shown in the following equation .

$$
\alpha=\left(\frac{n}{n-1}\right)\left(\frac{S_{t}^{2}-\sum V_{i}}{S_{t}^{2}}\right)
$$

In equation (1), $S_{t}^{2}$ is the total variance of the quantitative measures of impact indicators. $V_{i}$ is the quantitative variance between each quantitative impact indicator. Generally, the Crobach $\alpha$ coefficient value greater than 0.7 is considered high reliability, below 0.35 is considered low reliability, and 0.5 is the minimum acceptable level of reliability.

\subsection{Analyzing the impact of interior design on consumer behavior}

After organizing the user's questionnaire and preliminary data processing, LSTM-AE is used to analyze the user's consumption behavior to complete this study. LSTM-AE is an aspect-level LSTM algorithm based on the attention mechanism, and aspect information is still crucial when classifying the polarity of a given aspect of a sentence. If different aspects are considered, opposite polarities may be obtained. To make better use of aspect information, a vector is embedded here for each aspect in the learning. Let $H \in R^{d \times N}$ be a matrix consisting of hidden vectors $\left[h_{1}, \cdots, h_{N}\right]$ generated by the LSTM, where $d$ is the 
size of the hidden layer, $N$ is the length of the user behavior language expression statement, and $V_{a}$ denotes the aspect-level embedding. The state of the input word vector LSTM at each moment is updated by combining the hidden layer state and the aspect word vector, and the aspect words are trained together as parameters to obtain the weight representation of the sentence under a given aspect. The specific formula is as follows[13-14].

$$
\left\{\begin{array}{l}
M=\tanh \left(\left[\begin{array}{c}
W_{h} H \\
W_{v} v_{a} \otimes e_{N}
\end{array}\right]\right) \\
\alpha=\operatorname{soft} \max \left(w^{T} M\right) \\
r=H \alpha^{T} \\
M \in R^{\left(d+d_{a}\right) \times N}, r \in R^{d}
\end{array}\right.
$$

In equation (2), $r$ is a weighted representation of the sentence given the aspect. $M$ is the new hidden layer representation obtained by splicing the LSTM output with the aspect vector as input, after one layer of neural network. $w$ is the input subvector. $W$ is the input weight. $v_{a} \otimes e_{N}$ means that after stitching and copying into $\mathrm{N}$ copies $v_{a}$ [15].After processing the feedback survey content of theme restaurant users as mentioned above, we can determine the users' perceived feedback on different influencing factors.

Through the above detailed process, it is realized to study the influence of theme restaurant interior design on users' consumption behavior using the principle of interior environment space layout. Before using the influence research analysis method proposed above, detailed research should be conducted with different market contexts and consumption ability to obtain better analysis results.

\section{Experimental study and analysis}

This experiment is a simulated scenario experiment, and the experiment takes the form of comparing the impact analysis method mentioned in the literature [3] with the method proposed in the text. By collecting data, the more common theme restaurants in the market were selected as the reference for the simulation scenario of this experiment. The impact study of the actual theme restaurants was conducted using the two analysis methods separately, and the results were applied in different experimental scenarios. The feasibility of the method is judged by comparing the feedback content of users from different regions and age groups, as well as the revenue of the restaurants before and after the application of the two analysis methods.

Figure 2 below shows the revenue of the three restaurants during this simulation experiment. The bar graph in the figure shows the application of the method in the text and the line graph shows the application of the method in the literature.

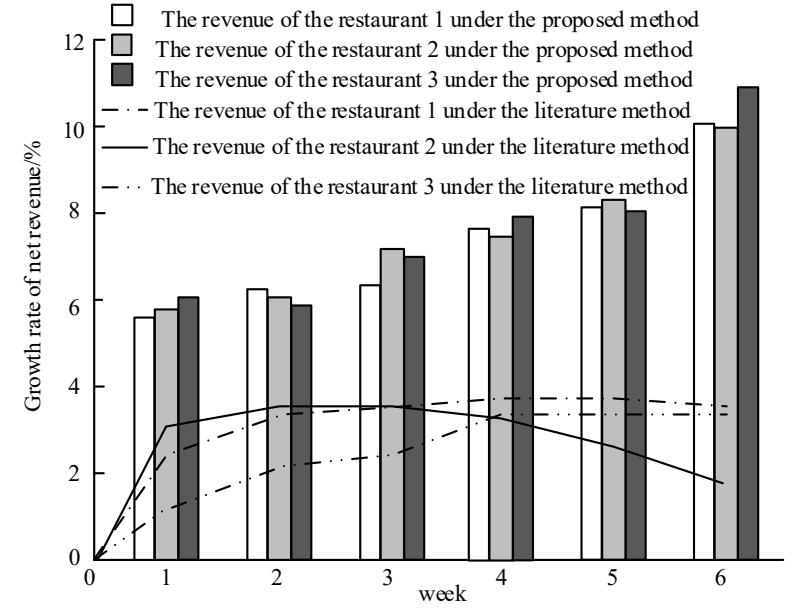

Figure 2 Comparison of restaurant revenue

Analyzing figure 2, the revenue of the three restaurants improved significantly after applying the method in the paper; applying the method in the literature [3], the restaurants had a significant increase in revenue at the beginning, but after a period of time both restaurants except restaurant 3 showed a significant decrease in revenue.

\section{Conclusion}

The rapid economic development in recent years has led to the improvement of people's living standards, and people's requirements for food and beverage have transitioned from the initial eating to the level of hygiene, nutrition, health, status and cultural consumption. Theme restaurants, a unique restaurant business model, emerged in the context of meeting the needs of customers at different levels of dining. The emergence of this theme restaurant meets the individual needs of consumers, spiritual experience, the consumer or the restaurant users simply dining behavior into a form of cultural consumption. This paper investigates the impact of interior design on user consumption behavior using theories related to the spatial layout of the interior environment, thus providing some theoretical support for future theme restaurant operations.

\section{References}

1. Duonamou Lucie, Konate Alexandre, Djègo Djossou Sylvie, et al. Consumer perceptions and reported wild and domestic meat and fish consumption behavior during the Ebola epidemic in Guinea, West Africa[J]. PeerJ, 2020, 8: e9229.

2. Inwon Kang, Ilhwan Ma. A Study on Bandwagon Consumption Behavior Based on Fear of Missing Out and Product Characteristics[J]. Sustainability, 2020, 12(6):2441.

3. Li Junwei, Wu Le, Hong Richang, et al. Random walk based distributed representation learning and prediction on Social Networking Services[J]. Information Sciences, 2021, 549 :328-346. 
4. Rachel E.N. Reyes, Alzahra J. Al Omran, Daryl L. Davies, et al. Antibiotic-induced disruption of commensal microbiome linked to increases in bingelike ethanol consumption behavior[J]. Brain Research, 2020, 1747:147067.

5. Johanna Palcu, Simona Haasova, Arnd Florack. Advertising models in the act of eating: How the depiction of different eating phases affects consumption desire and behavior[J]. Appetite, 2019, 139 :59-66.

6. Eric W.K. See-To, Eric W.T. Ngai. An empirical study of payment technologies, the psychology of consumption, and spending behavior in a retailing context[J]. Information \& Management, 2019,56(3): 329-342.

7. Muhammad Awais, Tanzila Samin, Muhammad Awais Gulzar, et al. Unfolding the Association between the Big Five, Frugality, E-Mavenism, and Sustainable Consumption Behavior[J]. Sustainability, 2020, 12(2):1-1.

8. Nigro Giovanna, Matarazzo Olimpia, Ciccarelli Maria, et al. To chase or not to chase: A study on the role of mentalization and alcohol consumption in chasing behavior.[J]. Journal of behavioral addictions, 2019, 8(4):743-753.

9. Costanza Nosi, Lamberto Zollo, Riccardo Rialti, et al. Sustainable consumption in organic food buying behavior: the case of quinoa[J]. British Food Journal, 2020, 122(3):976-994.

10. Flore Vallet, Benjamin Tyl. Implementation of an eco-innovation toolbox to stimulate design teams: A case of interior design[J]. Procedia CIRP, 2020, $90: 334-338$.

11. Zhu Anzhi. Application of artificial intelligence technology and embedded digital image in interior design[J]. Microprocessors and Microsystems, 2021, $81: 103782$.

12. Khorawan Ngamvoratham, Thanin Ratanaolarn, Jitjayang Yamabhai, et al. Interior Environmental Design for a Leisure Learning Area in the National Gallery of Thailand[J]. Mediterranean Journal of Social Sciences, 2019, 10(2):99-112.

13. Wong C H, Aziz A A . Perceptions of Youngsters on Interior Space Quality in Relation to Materiality and Spatial Design[J]. International Journal of Built Environment and Sustainability, 2020, 8(1):103-119.

14. Chung, Miryum. Nursing Home Environmental Perception and Preference Differences between Generations[J]. Journal of the Korean Institute of Interior Design, 2019, 28(1) :188-198.

15. Rila Anggraeni, Dimas Hendrawan, Yu-Wen Huang. The Impact of Theme Restaurant Servicescape on Consumer' s Value and Purchase Intention[P]. 23rd Asian Forum of Business Education(AFBE 2019), 2020, 144, 226-232.

16. Pooja Ahuja, Pankaj Tiwari. Examining Consumer Loyalty, Internet of Things (IoT), and Theme Restaurants in the Delhi Region: An Empirical
Study[J]. International Journal of Knowledge-Based Organizations (IJKBO), 2020, 10(4):1-12. 